\title{
Thermal Expansion of Five Selected Optical Glasses
}

\author{
Peter Hidnert
}

\begin{abstract}
Coefficients of linear thermal expansion of five optical glasses were determined by a strain gage method for the range between room temperature and $60^{\circ} \mathrm{C}$. The coefficients of expansion of two crown glasses are $7.6 \times 10^{-6}$ per deg C. A rare-earth glass was found to have a slightly lower coefficient of expansion, but the coefficients of expansion of two flint glasses are 10 percent larger than the other glasses.
\end{abstract}

This paper gives coefficients of linear thermal expansion of five samples of optical glasses. The chemical composition of these glasses are given in Research Paper 2504.

The diameter of each sample of glass was $30 \mathrm{~mm}$. The length of each sample was $105 \mathrm{~mm}$, except sample DF (table 1), which was $97 \mathrm{~mm}$ long. Determinations were made on the linear thermal expansion of these glasses on heating and cooling between $23^{\circ}$ and $60^{\circ} \mathrm{C}$. An electric-resistance strain-gage method was used. When a strain gage, consisting of a fine wire, is attached to a sample of solid material, changes in length of the wire accompanying thermal expansion of the sample are proportional to the changes in electrical resistance of the wire. Information about commercially available types of wire strain gages has been given by Ramberg. ${ }^{2}$

Two SR4 (type A 11) strain gages connected in series, were attached with a cellulose nitrate cement on opposite sides of each sample of glass and of each of three reference samples. These reference samples were duplicates of samples of invar, glass, and steel on which coefficients of linear thermal expansion had previously been determined by the precision micrometric method ${ }^{3}$ of the Bureau. After the cement was sufficiently dried, the cemented gages were coated with wax. The glass and reference samples with their attached gages were then subjected to two cycles of heating and cooling between room temperature and $60^{\circ} \mathrm{C}$, in order to allow the gages to adjust themselves to the samples before observations were made. During these cycles of heating and cooling, all samples were held at the maximum temperature for 2 hours.

The samples of glass and the reference samples with attached strain gages were supported vertically in an electrically controlled temperature cabinet. Two copper leads extended from each sample and from each reference sample to an SR4 strain indi-

\footnotetext{
1 The companion papers in this issue present values of the index of refraction. spectral transmissive properties, and densities for five different types of optical glasses produced by the Optical Glass Section of the National Bureau of Standards. These measurements have been made in the different organizational parts of the Bureau appropriate to each type of measurement, and are, therefore, of the Bureau appropriate to each type of measurement, and are, therefore,
presented separately. For each type of glass the samples have been taken from presented separately. For each type of glass the samples have been taken from the same melt, and, therefore, these
set of data for the five glasses studied.

2 W. Ramberg, Contributions of electricity to mechanics, J. Wash. A cad. Sci. 39, 281 (Sept. 1949).

39, 281 (Sept. 1949). H. Souder, Thermal expansion of solids, NBS Circular 486 (1950).
}

cator (type K). Four copper-constantan thermocouples placed symmetrically in the region of the glass samples and the reference samples were connected to a precision potentiometer.

Observations were made at room temperature and at $60^{\circ} \mathrm{C}$, during three cycles of heating and cooling, on each sample of glass in conjunction with each reference sample of known expansion. These observations at constant temperatures, consisted of strainindicator readings recorded to the nearest microinch per inch and of potentiometer readings recorded to the nearest microvolt. The latter readings were converted to temperatures to the nearest $0.1 \mathrm{deg}$ C. The coefficients of linear thermal expansion of the five samples of glass given in table 1, were derived from the data. These coefficients of expansion are believed to be accurate to $\pm 0.1 \times 10^{-6}$. TABLE 1. Coefficients of linear thermal expansion of some
optical glasses

\begin{tabular}{|c|c|c|}
\hline Glass a & $\begin{array}{c}\text { Observed aver- } \\
\text { age coefficient } \\
\text { of expansion per } \\
\text { deg C between } \\
23^{\circ} \text { and } 60^{\circ} \mathrm{C}\end{array}$ & $\begin{array}{c}\text { Computed b } \\
\text { coefficient of } \\
\text { expansion per } \\
\text { deg C }\end{array}$ \\
\hline BSC, $n=1.517, \nu=64.5$ & $7.6 \times 10^{-6}$ & $7.6 \times 10^{-6}$ \\
LBC, $n=1.574, \nu=57.7$ & 7.6 & 7.8 \\
F $, n=1.620, \nu=36.2$ & 8.4 & 8.5 \\
DF $, n=1.649, \nu=33.8$ & 8.3 & 8.5 \\
Rare earth, $n=1.673, \nu=56.2$ & 7.5 & $($ c) \\
\hline
\end{tabular}

s $n=$ index of refraction for the sodium $D$ line, and $\nu$, the $A$ bbe value $=(n-1) /$ $\left(n_{F}-n_{C}\right)$, where $n_{F}$ and $n_{C}$ are refractive indices for the hydrogen $F$ and $C$ lines,

respectively. to $90^{\circ} \mathrm{C}$ (see footnote 4 ).

- $\mathrm{N}_{0}$ factor was given by English and Turner for $\mathrm{La}_{2} \mathrm{O}_{3}$ in this glass.

The last column in table 1 gives coefficients of expansion computed from English and Turner's ${ }^{4}$ factors. ${ }^{5}$ One-third of the sum of the products of the factors and the precentages of the corresponding constituent oxides of a glass gives a computed coefficient of linear thermal expansion. The agreement between the observed and computed coefficients of linear expansion of the glasses is good.

${ }^{4}$ S. English and W. E. S. Turner, Relationship between chemical composition and the thermal expansion of glasses, J. Am. Ceram. Soc. 10, 551 (1927); Corrections on "Relationship between chemical composition and the thermal expansion of glasses," J. Am. Ceram. Soc. 12, 760 (1929).

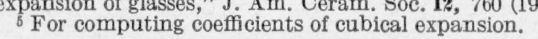


The coefficients of expansion of the two crown glasses (BSC 1.517 and LBC 1.574) and the rare earth glass 1.673 are practically the same and about 10 percent smaller than the coefficients of expansion of the two flint glasses (F 1.620 and DF 1.649).
The author acknowledges his indebtedness to R. C. Valore, Jr. of the NBS Structural Engineering Section for helpful suggestions in the application of the strain-gage method and to H. S. Krider of the Length Section for assistance in the experimental work.

Washington, July 14, 1953. 